only 2 per cent of the currently accepted age of the Universe or it would have accreted more than its own mass. As approximately 2 per cent of all galaxies are active, the new evidence could suggest that all galaxies have an active phase for 2 per cent of their life times.

Much argument about $\mathrm{X}$ rays from active galaxies centres on the radiation mechanism involved. Is it thermal? Or is it due to very high-energy relativistic electrons (like those found in radio galaxies)? Such high-energy electrons must lose their energy extremely fast. Tennant $e t$ al. conclude that for such non-thermal mechanisms to be viable, some process must continuously re-accelerate electrons throughout the emitting volume. Are thermal processes simpler? Not really; the $\mathrm{X}$-ray spectrum is consistent with optically thin thermal emission, but from such a small volume, thermal radiation should be optically thick. Thermal processes are only viable in more sophisticated models where very hot electrons give a 'boost' to UV photons inserted from outside the X-rayemitting region.

Why have such rapid fireworks not been seen from other objects? In another, as yet unpublished, investigation ${ }^{10}$, Tennant $e t$ al. find that of 30 objects studied, only NGC 6814 showed such behaviour. In other studies of Seyfert galaxies, only NGC 4051 and possibly NGC 3227 have shown variability on a time scale of minutes ${ }^{11,12}$. All three galaxies are rather low-luminosity examples of the Seyfert phenomenon, but other low-luminosity objects have not shown very rapid variations. There are indications that these objects have anomalous optical and IR properties ${ }^{13}$, but the data are still scant and confusing.

Recently preprinted observations with the Einstein Observatory ${ }^{14}$ have claimed $100 \mathrm{~s}$ variability in the quasar $1525+227$. The data are not as convincing as in other cases, but if confirmed, the new observations could be of great importance; the X-ray luminosity of $1525+227$ is several hundred times that of NGC 6814 , and the constraint placed on models would be correspondingly tighter. Hope for further progress in these matters in the near future rests on the performance of the European X-ray satellite EXOSAT, due to be launched in autumn 1982.

1. Tennant, A. F., Mushotzky, R. F., Boldt, E. A. \& Swank J. H. Astrophys. J. 251, 15 (1982).

2. Burbridge, E. M. A. Rev. Astr. Astrophys. 5, 399 (1967).

3. Elvis, M. Mon. Not, R, astr. Soc. 177, $7 \mathrm{p}$ (1976).

4. Winkler, P. F. \& White, A. E. Astrophys. J. Lett. 199 L139 (1975)

5. Mushotzky, R. F., Hott, S. S. \& Serlemitsos, P. J. Astrophys. J. Lett. 225, L115 (1978).

6. Delvaille, J. P., Epstein, A. \& Schnopper, W. H Astrophys. J. Lett. 219, L81 (1978).

7. Lawrence, A. Mon. Not, R astr. Soc. 192, 83 (1980).

8. Marshall, N., Warwick, R. S. \& Pounds, K. A. Mon Not. R. astr. Soc. 194, 987 (1981)

9. Tananbaum, H. et al. Astrophys. J. 223, 74 (1978).

10. Tennant, A. F. et al. (in preparation).

11. Marshall, F. E., Becker, R. H., Holt, S. S. \& Mushotzky, R. F. Bull. Am. astr. Soc. 12, $796(1980)$

12. Mushotzky, R. F. Private Communication.

13. Lawrence, A. \& Elvis, E. Astrophys. J. (in the press)

14. Matilsky, T., Schrader, C. \& Tananbaum, H. Preprint (1982).

\section{Receptors for T-cell growth}

\author{
from D.E. Kipp and B.A. Askonas
}

THE study of lymphocyte mediators has recently become an active area of cellular immunology. Although it has been known for more than twenty years that conditioned media from lectin-stimulated spleen cells contain lymphokines which enhance in vivo immune responses ${ }^{1,2}$, the more recent discovery that conditioned media can support the continuous growth of activated $\mathrm{T}$ cells in vitro ${ }^{3}$ has made the $\mathrm{T}$-cell growth factor(s) (TCGF) of particular significance.

In vivo, the importance of TCGF lies in the control of $\mathrm{T}$-cell clonal expansion and responses. In vitro, the use of TCGF has proved of enormous value since it has made it possible to select and grow T-cell clones with different functions, such as cytotoxic $\mathrm{T}$ cells or T-helper cells. For the first time, single clones of $\mathrm{T}$-cell subpopulations of known antigen specificity can be studied. This has made it possible to characterize their phenotype and functions, and their release of various lymphokines, factors, or interferon; to type lymphocyte-defined human histocompatibility antigens; and to determine the fine specificity of individual $T$ cells and their recognition of antigen in conjunction with products of the major histocompatibility gene complex. It has also made it possible to provide important sources of lymphokines.

The resting $T$ cell is unresponsive to TCGF and presumably does not express receptors for TCGF on its cell surface. In contrast, mitogen- or antigen-activated $\mathrm{T}$-cells respond to $\mathrm{TCGF}^{4}$ and continue to grow in vitro. In a recent report, R.J. Robb, A. Munck and K.A. Smith ${ }^{5}$ define receptors for TCGF by examining the binding of biosynthetically labelled TCGF derived from a human T-leukaemia cell line (JURKAT) to various cell types. TCGF was purified after incubation of JURKAT cells with radiolabelled amino acids to show a single band on SDSpolyacrylamide gel electrophoresis at a molecular weight of about 15,000, equivalent to the previously estimated molecular size of human TCGF ${ }^{6}$. This was the only measure of purity, but the material was functionally active and its binding properties to cells paralleled functional activity. The authors estimated by equilibrium binding assays of the purified radiolabelled TCGF that there were $10-15,000$ receptors on TCGF-dependent mouse cytotoxic or helper T-cell clones. Furthermore, while normal murine splenocytes and thymocytes as well as human peripheral blood cells showed just barely detectable binding of radiolabelled TCGF, alloantigen- or lectin-stimulated cells had

D.E. Kipp and B.A. Askonas are in the Immunology Division of the MRC National Institute of Medical Research, Mill Hill, London NW7 IAA. easily detectable receptors. This result supports the observations that only antigen-or mitogen-activated $\mathrm{T}$ cells are responsive to TCGF, whereas unstimulated $\mathrm{T}$ cells (and lipopolysaccharideactivated $B$ cells) are not reactive to TCGF and appear not to possess any receptors for TCGF.

Robb et al. also studied the binding of radiolabelled TCGF to a variety of human neoplastic cell lines. All of the non-T-cell lines and 90 per cent of human $\mathrm{T}$-cell lines failed to bind TCGF. The human T-cell lines have been classed as T-cell lines on the basis of rosette formation with sheep red blood cells and this may not be an ideal criterion. The absence of TCGF receptors may therefore be explained in several ways. The transformed cell lines may have lost expression of their receptors or may have been derived from early $T$ cells that had not yet expressed receptors. Certainly, antigendependent untransformed $T$ cells, be they helper or cytotoxic cells, can respond to $\mathrm{TCGF}^{7,8}$. The one human $\mathrm{T}$-cell line found to bind TCGF, which apparently grows well without the addition of exogenous TCGF, has recently been shown to both produce and respond to TCGF'.

The work of Robb et al. also confirmed the specificity of the human receptor for TCGF. The binding of radiolabelled human TCGF to its receptor was not inhibited by any other growth factor tested or by TCGF derived from rodent spleen cells which does not act on human $\mathrm{T}$ cells. On the other hand-human TCGF is not species specific and acts on rodent $T$ cells as well ${ }^{10}$. The association and dissociation of human TCGF binding to mouse cytotoxic $T$ cells showed a rapid turnover of TCGF and its receptor as well as a rapid degradation of TCGF after association with its receptor. This rapid degradation may have an important regulatory significance as the presence of TCGF would allow for the continued expansion of activated $\mathrm{T}$ cells in the lymphoid tissues, and thus block any dampening of the immune response when it is no longer needed.

These studies provide insight into the regulation of the immune response by TCGF. However, it needs to be stressed that this is only the beginning of such studies. Clearly it will be important to define the nature of the receptor for TCGF and we thus await eagerly the next chapter of this unfolding story.

\footnotetext{
Gordon, J. \& MacLean, L.D. Nature 208, 795 (1965). . Kasakuro, S \& Lowenstin. Nature 208, 894 (1965).

3. Morgan, D.A., et al. Science 193, 1007 (1976).

3. Morgan, D.A., et al. Science 193, 1007 (1976).

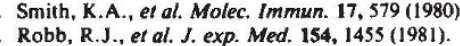

Watson, J. et al. J. exp. Med. 150, 849 (1979).

Watson, J. J. exp. Med. 150, 1510 (1979).

. Gillis, S. \& Smith, K.A. Nature 268, 154 (1977).

Gootenberg, J.E. et al. J. exp. Med. 154, 1403 (1981)

10. Gillis, S. et al. J. Immun. 120, 2027 (1978)
} 